\title{
A toxina botulínica na correção de sorriso gengival: uma revisão de literatura
}

\section{Botulinum toxin in the correction of gingival smile: a literature review}

DOI: $10.46919 / \operatorname{archv1n1-004}$

Recebimento dos originais: 01/01/2020

Aceitação para publicação: 10/01/2020

\author{
Yali Carvalho Ribeiro de Castro \\ Graduada em Odontologia pela Universidade Tuiuti do Paraná \\ Instituição: Universidade Tuiuti do Paraná- UTP \\ Endereço: Rua Sydnei Antonio Rangel Santos, 238, bairro Santo Inácio - Curitiba, Paraná, CEP \\ 82010-330 \\ E-mail: yalirc@hotmail.com \\ Patrícia Marcoccia de Souza \\ Mestre em Dentística Restauradora pelo Centro de Pesquisas Odontológicas São Leopoldo Mandic \\ Instituição: Universidade Tuiuti do Paraná- UTP \\ Endereço: Rua Sydnei Antonio Rangel Santos, 238, bairro Santo Inácio - Curitiba, Paraná, CEP \\ 82010-330 \\ E-mail: patriciagrude@hotmail.com
}

\section{RESUMO}

INTRODUÇÃO: Um sorriso gengival (SG) afeta a estética e o estado psicológico, pois geralmente diminui a auto-confiança levando a esconder ou controlar o sorriso. Um sorriso com mais de $2 \mathrm{~mm}$ de gengiva exposta é chamado sorriso gengival. Ele pode ser devido à uma ou mais dasseguintes etiologias: erupção passiva de dentes, extrusão dento alveolar, excesso vertical maxilar e músculos dos lábios curtos ou hiperativos. O tratamento de sorriso gengival deve ser previsto de acordo com a sua causa (s).

METODOLOGIA: Trata-se de uma revisão de literatura baseada no uso da toxina botulínica, um método novo, eficiente e reversível para tratamento de sorriso gengival.

CONCLUSÃO: A toxina botulínica tem sido considerada um tratamento de mínima invasão, mais conservadora, mais eficaz, mais rápida, mais segura em comparação aos procedimentos cirúrgicos.

Palavras-chave: Sorriso gengival; toxina botulínica; estética; cirurgião dentista

\begin{abstract}
INTRODUCTION: A gingival smile (SG) affects aesthetics and psychological status, as it generally decreases self-confidence leading to hiding or controlling the smile. A smile with more than $2 \mathrm{~mm}$ of exposed gum is called a gum smile. It can be due to one or more of the following etiologies: passive tooth eruption, tooth extrusion, vertical maxillary excess and short or hyperactive lip muscles. The treatment of gingival smile should be provided according to its cause (s).
\end{abstract}


METHODOLOGY: This is a literature review based on the use of botulinum toxin, a new, efficient and reversible method for the treatment of gingival smile.

CONCLUSION: Botulinum toxin has been considered a minimally invasive, more conservative, more effective, faster, safer treatment compared to surgical procedures.

Keywords: Gingival smile; botulinum toxin; aesthetics; dental surgeon.

\section{INTRODUÇÃO}

O sorriso é provavelmente a expressão facial mais agradável e significativa de uma pessoa. Em 1974, Rubin categorizados 3 tipos de sorriso. Um sorriso “Mona Lisa” é dominado pelo grande músculo zigomático e representado por cantos acentuadamente elevados. Um "sorriso canino" é caracterizado por uma elevação sobre a porção média do lábio superior através da ação de um músculo elevador proeminente do lábio superior. E o sorriso "full dentadura" é dominado por todos os músculos retratores superiores, além dos depressores menores, resultando em um sorriso que expõe todos os dentes (SUBER et al., 2014).

Em termos de incidência, aproximadamente 7\% dos homens e 14\% das mulheres têm exposição gengival excessiva com um sorriso. A exposição excessiva gengival, ou "sorriso gengival", é definido como a exibição de $2 \mathrm{~mm}$ ou mais da mostra gengival ao sorrir. Um sorriso gengival pode ser classificado como anterior, posterior, misto, ou assimétricos, dependendo da área de tecido gengival excessiva demonstrada. As causas incluem erupção passiva dental atrasada, excesso vertical da maxila, e / ou músculo elevador do lábio superior hiperfuncional (SUBER et al., 2014).

Para lidar com essas causas, abordagens anteriores incluíram gengivoplastia, tratamento ortodôntico, cirurgia ortognática, a ressecção óssea e miotomia ou miectomia dos músculos faciais específicas (SUBER et al., 2014).

A assimetria facial tem uma forte correlação com domínios subjetivos como prejuízo na interação social e percepção de auto-imagem e aparência. injeções de toxina botulínica representam uma técnica minimamente invasiva que é útil na restauração da simetria facial em repouso e durante o movimento. A toxina botulínica em combinação com a terapia física pode ser particularmente útil (COOPER, LUI e NDUKA, 2017).

A toxina botulínica (TB) é uma neurotoxina potente produzida pela bactéria Clostridium botulinum. Sete formas distintas (TB-A, B, C, D, E, F e G) foram descritas, com TB-A e TB-B disponíveis comercialmente (FRANÇA et al., 2017). A TB bloqueia a liberação de acetilcolina e vários 
outros neurotransmissores das vesículas pré-sinápticas, desativando as proteínas SNARE e tem uma longa história de aplicação terapêutica em condições neurológicas com um forte perfil de eficácia e segurança. Como é sabido, a pele interage com o sistema nervoso; o sistema neurológico participa diretamente da inflamação cutânea e da cicatrização de feridas (KIM, HONG e KIM, 2017).

Objetivo: Avaliar o grau de eficiência da toxina botulínica para exposição excessiva de gengiva na maxila ou o chamado sorriso gengival.

\section{METODOLOGIA}

Trata-se de uma revisão de literatura para conclusão de curso, baseada no uso da toxina botulínica, um método novo, eficiente e reversível para tratamento de sorriso gengival. Foram incluídas neste estudo as publicações dos anos 2014 a 2019. Para construção da revisão foram utilizadas as seguintes bases de dados: Scielo, Lilacs, Pubmed e Google acadêmico. As palavras-chaves utilizadas para pesquisa foram: sorriso gengival, toxina botulínica, estética e cirurgião dentista. Os idiomas dos artigos: Espanhol, Inglês e Português.

\section{DISCUSSÃO}

No decorrer dos últimos anos muitas pessoas vêm buscando cada vez mais por um padrão de beleza imposto pela sociedade, esses padrões não estão relacionados apenas ao corpo como também à beleza orofacial. É inegável, no entanto, que a forma de envelhecimento celular ocorre de forma natural com o aumento da idade. A expectativa de vida subiu grandemente durante as últimas décadas, provocando assim uma maior preocupação com a aparência física. Diante desse motivo, muitos submetem-se a procedimentos estéticos com o objetivo de diminuir os efeitos adversos trazidos com o tempo relata Srivastava; Kharbanda e Shah (2015).

Srivastava; Kharbanda e Shah (2015) em concordância com Garbin et al. (2019) descrevem que, atualmente, a Harmonização Facial vem sendo cada vez mais desempenhada pelos Cirurgiões-Dentistas, e por meio de novas técnicas, há grande perspectiva em harmonizar a face de maneira mais suavizada e natural, deixando os resultados, os mais discretos possíveis. Respeitando a idade, com o conhecimento de visagismo e anatomia da face de cada paciente, tais técnicas atendem assim, os resultados esperados pelos pacientes, na tentativa de minimizar as marcas do envelhecimento. O Cirurgião-Dentista (CD), mediante licenciamento para atuação através de residências ou especializações, são capazes de praticar tais procedimentos, já que essa área específica envolve um processo multidisciplinar como citado por Brasil (2016). 
Para Yesilbek; Simsek e Valério (2016) é indispensável compreender a importância das intervenções precoces no tratamento das desarmonias faciais, pois proporciona aos pacientes crianças e adolescentes uma melhor qualidade de vida não só no aspecto funcional, mas principalmente no aspecto psicossocial.

O sorriso de uma pessoa depende de três componentes principais: dentes, gengivas e lábios, muitos estudaram os componentes de um sorriso atraente, estudos como Suber et al. (2014) e Nasr et al. (2015) concluíram que o sorriso gengival é definido com $2 \mathrm{~mm}$ ou mais de exposição gengival. Logo, os autores Nunes et al. (2014) e AI-fouzan et al. (2017) consideraram sorriso gengival, os pacientes que apresentam uma exposição superior a $3 \mathrm{~mm}$ de tecido gengival.

Para Dym e Pierre II (2019) a avaliação completa do perfil facial do paciente pode fornecer informações uteis para ajudar a identificar a causa do sorriso gengival. Trabalhos como o de Nasr et al. (2015) e Chagas et al. (2018), relatam que o sorriso gengival pode ser resultado de uma erupção passiva atrasada, um excesso vertical maxilar, ou hiperatividade dos músculos elevadores do lábio superior.

As modalidades de tratamento para o sorriso gengival incluem o tratamento ortodôntico, ressecção óssea, gengivoplastia, reposicionamento labial e cirúrgico. Dentro das formas de tratamento, a injeção de toxina botulínica (TB) é uma técnica simples, conservadora e eficaz para o tratamento de sorriso gengival causado por músculos hiperfuncionais do elevador do lábio superior, no entanto, esse efeito é transitório, concordam os escritores Pedron e Mangano (2018) e Bertossi et al. (2018).

Ainda segundo Bertossi et al. (2018) é sugerido para tratar o sorriso gengival com três locais de injeção de toxina, para o músculo elevador lábio superior e asa do nariz e um central para o depressor do septo nasal. Todavia, os autores Srivastava; Kharbanda e Shah (2015) defendem um ponto de injeção de TB conhecido como "ponto yonsei" (centro do triângulo: levantador do lábio superior e asa do nariz e zigomático menor).

Os autores Srivastava; Kharbanda e Shah (2015), Dressler (2015) e Delpachitra; Sklavos e Dastaran (2018) comentam que as doses de TB utilizadas no tratamento dependem da marca e preparação específica, pois a unidade de um produto não é a mesma que a outra. A toxina botulínica está em uso clínico desde 1967 e os dois tipos disponíveis mais comuns são o Botox e Dysport.

É visto por Kim; Hong e Kim (2017), Cooper; Lui e Nduka (2017) e Pedron e Mangano (2018) que a toxina botulínica é uma neurotoxina produzida pela bactéria Clostridium botulinum e é descrita em sete formas distintas (BTX-A, B, C, D, E, F e G). A TB trabalha bloqueando a liberação de acetilcolina e 
vários outros neurotransmissores das vesículas pré-sinápticas, desativando as proteínas SNARE e tem uma longa história de aplicação terapêutica em condições neurológicas com um forte perfil de eficácia e segurança.

Com relação aos efeitos adversos, escritores como Srivastava; Kharbanda e Shah (2015), Cooper; Lui e Nduka (2017) e Moreira et al. (2019) concordam que o botulinum é uma das toxinas mais letais conhecidas, porém, o uso da TB é considerado um procedimento seguro, no entanto, pode causar possíveis complicações como reação alérgica, eritema, dor e edema no local. Logo, em contraposição, Heikkila et al. (2018) obtiveram resultados que a toxina pode se espalhar a partir da articulação, mas o seu impacto clínico é baixo.

As contraindicações apontadas pelos autores Srivastava; Kharbanda e Shah (2015) incluem alergia a qualquer um dos constituintes da TB-A ou B, infecção no local, gravidez e lactação, pacientes que recebem tratamentos que interferem na transmissão neuromuscular ou relaxantes musculares, pois o efeito pode ser potencializado.

Visto isso, autores como Pedron e Mangano (2018), Duruel et al. (2019), Duruel; Ataman-Duruel; Berker e Toezuem (2019) concluem que a aplicação da toxina botulínica é uma alternativa menos invasiva, mais rápida, mais segura e mais eficaz.

\section{CONCLUSÃO}

É importante avaliar as expectativas estéticas dos pacientes e mostrar as possíveis soluções terapêuticas. A toxina botulínica é considerada como uma modalidade minimamente invasiva, rápida e acessível, que pode substituir procedimentos cirúrgicos extensos para correções de sorriso gengival.

\section{REFERÊNCIAS}

AL-FOUZAN, A. F. et al. Botulinum Toxin for the Treatment of Gummv Smile. J Contemp Dent Pract, v.18, n.6, p.474-478, 2017.

BERTOSSI, D. et al. Italian consensus report on the aesthetic use of onabotulinum toxin A. J Cosmet Dermatol, v. 17, n. 5, p. 719-730, 2018.

BRASIL. Conselho Federal de Odontologia (CFO). Resolução n 176 de 6 set. 2016. Revoga as Resoluções CFO-112/2011, 145/2014 e 146/2014, referentes à utilização da toxina botulínica e preenchedores faciais, e aprova outra em substituição. Disponível em: $<$ http://www.lex.com.br/legis_27187904_RESOLUCAO_N_176_DE_SETEMBRO_DE_2016.aspx>. Acesso em: 27 mar, 2020 
CHAGAS, T. F. et al. Duration of effectiveness of Botulinum toxin type A in excessive gingival display: a systematic review and meta-analysis. Braz Oral Res, v. 32, 2018.

COOPER, L.; LUI, M.; NDUKA, C. Botulinum toxin treatment for facial palsy: a systematic review. J Plast Reconstr Aesthet Surg, v. 70, n. 6, p. 833-841, 2017.

DELPACHITRA, S. N.; SKLAVOS, A. W.; DASTARAN, M. Clinical uses of botulinum toxin A in smile aesthetic modification. Br Dent J, v. 225, n. 6, p. 502-506, 2018.

DRESSLER, D. Botulinum toxin drugs: brief history and outlook. J Neural Transm, v. 123, n. 3, p. 277 279, 2016.

DURUEL, O. et al. Ideal Dose and Injection Site for Gummy Smile Treatment with Botulinum Toxin-A: A Systematic Review and Introduction of a Case Study. Int J Periodontics Restorative Dent, v. 39, n. 4, 2019.

DURUEL, O. et al. Treatment of Various Types of Gummy Smile With Botulinum Toxin-A. J Craniofac Surg, v. 30, n. 3, p. 876-878, 2019.

DYM, H.; PIERRE, R. Diagnosis and Treatment Approaches to a "Gummy Smile". Dent Clin North Am, v. 64 , n. 2, p. 341-349, 2020.

FRANÇA, K. et al. The history of Botulinum toxin: from poison to beauty. Wien Med Wochenschr, v. 167, n. 1, p. 46-48, 2017.

GARBIN, A. J. I. et al. Orofacial Harmonization and its Implications in Dentistry. Braz J Surg Clin Res, v. 27, n.2, p. 116-122, 2019.

HEIKKILÄ, H. M. et al. Assessing adverse effects of intra-articular botulinum toxin A in healthy Beagle dogs: A placebo-controlled, blinded, randomized trial. PloS one, v. 13, n. 1, 2018.

KIM, Y. S.; HONG, E. S.; KIM, H. S. Botulinum toxin in the field of dermatology: novel indications. Toxins (Basel), v. 9, n. 12, p. 403, 2017.

MAZZUCO, R.; HEXSEL, D. Gummy smile and botulinum toxin: a new approach based on the gingival exposure area. J Am Acad Dermatol, v. 63, n. 6, p. 1042-1051, 2010.

MOREIRA, D. C. et al. Aplicação da toxina botulínica tipo A em sorriso gengival: relato de caso. Rev Gaúcha Odontol, v. 67, 2019.

NASR, M. W. et al. Botulinum toxin for the treatment of excessive gingival display: a systematic review. Aesthet Surg J, v. 36, n. 1, p. 82-88, 2016.

NUNES, L. et al. Tratamiento de la sonrisa gingival con la toxina botulínica tipo A: caso clínico. Rev Esp Cirug Oral y Maxilofac, v. 37, n. 4, p. 229-232, 2015.

PEDRON, I. G.; MANGANO, A. Gummy Smile Correction Using Botulinum Toxin With Respective Gingival Surgery. J Dent, v. 19, n. 3, p. 248, 2018.

SRIVASTAVA, S. et al. Applications of botulinum toxin in dentistry: A comprehensive review. Natl J Maxillofac Surg, v. 6, n. 2, p. 152, 2015.

SUBER, J. S. et al. OnabotulinumtoxinA for the treatment of a "gummy smile". Aesthet Surg J, v. 34, n. 3, p. 432-437, 2014. 
YESILBEK, B.; SIMSEK, S.; VALÉRIO, P. O impacto psicossocial da estética facial em crianças e adolescentes e a possibilidade de intervenções precoces: relato de dois casos clínicos. Rev Assoc Paul Cir Dent, v. 70, n. 2, p. 192-197, 2016.

SAMPAIO, C. A. C.; ALVES, F. K.; FALK, V. C. V. Arranjo socioprodutivo de base comunitária: Interconectando o turismo comunitário com redes de Comércio justo. Turismo Visão e Ação, v. 10, n 2. p. 244-262, 2008.

SINGER, P. Introdução à economia solidária. $3^{\text {a }}$ ed. São Paulo: Fundação Perseu Abramo, 2002. 